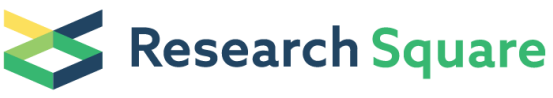 \\ Preprints are preliminary reports that have not undergone peer review. \\ They should not be considered conclusive, used to inform clinical practice, \\ or referenced by the media as validated information.
}

\section{Frequency and Clinical Impact of WT1 Mutations in the Context of CEBPA-Mutated Acute Myeloid Leukemia}

\author{
Ting Wang \\ Affiliated Changzhou Second Hospital of Nanjing Medical University \\ Haiying Hua \\ Wuxi Third people's hospital \\ Zhen Wang \\ the First Affiliated Hospital of Soochow University, Soochow University \\ Biao Wang \\ The Third Affiliated Hospital of Soochow University \\ Liujun Cao \\ Affiliated Jintan People's Hospital of Jiangsu University \\ Wei Qin \\ Affiliated Changzhou Second Hospital of Nanjing Medical University \\ Pin Wu \\ Wuxi Second people's hospital \\ Hongying Chao ( $\square$ chaohy2006@126.com ) \\ Affiliated Changzhou Second Hospital of Nanjing Medical University \\ XuZhang Lu \\ Affiliated Changzhou Second Hospital of Nanjing Medical University
}

Research Article

Keywords: Acute myeloid leukemia, WT1, CEBPA, mutation

Posted Date: May 27th, 2021

DOI: https://doi.org/10.21203/rs.3.rs-526446/v1

License: (c) (i) This work is licensed under a Creative Commons Attribution 4.0 International License. Read Full License 


\section{Abstract}

Introduction: Several studies have confirmed that mutations in the Wilms tumor 1 (WT1) gene occur in adult acute myeloid leukemia (AML). However, few data are available regarding the incidence of WT1 mutations in CEBPA ${ }^{\text {mut }}$ AML and their impact.

Methods: We retrospectively analyzed the frequency and clinical impact of WT1 mutations in 220 newly diagnosed AML patients with CEBPA mutations. Chromosome karyotype analysis was performed by $\mathrm{R}$ or $\mathrm{G}$ banding method and further confirmed either by fluorescence in situ hybridization (FISH) and/or by multiple reverse transcription polymerase chain reaction (multiple RT-PCR). Mutations were detected with a panel of 112 mutational genes using next-generation sequencing (NGS). FLT3-ITD, NPM1 and CEBPA mutation were detected by PCR Sanger sequencing.

Results: Overall, 30 WT1 mutations were detected in 29 of the 220 patients (13.18\%) screened. These mutations clustered overwhelmingly in exon 7 (16 mutations in 15 patients), but they were also detected in exon $8(n=6)$ and exon $9(n=8)$. WT1mutated (WT1 ${ }^{\text {mut }}$ ) patients presented with lower platelet counts $(P=0.0481)$, higher WT1 expression $(P=0.0371)$, and a negative trend with the M5 subtype ( $P=0.07)$ compared to WT wild-type $\left(W T^{\text {wt }}\right)$ patients. WT1 mutations were found to be significantly more frequent in AML patients with double-mutated CEBPA (CEBPA $\left.{ }^{\mathrm{dm}}\right)$ than in AML patients with single-mutated CEBPA $(P=0.043)$. Of note, the concomitant mutations in epigenetic regulators were inversely correlated with WT1 mutations $(P=0.003)$. Patients with newly diagnosed WT1 ${ }^{\text {mut }} A M L$ had inferior relapse-free survival, event-free survival and overall survival compared with patients with $\mathrm{WT} 1^{\mathrm{wt}} \mathrm{AML}(P=0.002,0.004$, and 0.010 , respectively).

Conclusion: Our data showed that WT1 mutations are frequently identified in CEBPA ${ }^{\text {mut }}$ AML, especially in CEBPA ${ }^{\text {dm }}$ AML. Patients with WT1 mutations show distinct clinical features, a spectrum of comutations, and poor prognosis compared to WT1 wild-type patients.

\section{Introduction}

CCAAT/enhancer binding protein a (CEBPA) plays a crucial role in the repression of self-renewal, cell cycle arrest, and myeloid differentiation of mature neutrophils during normal hematopoiesis[1, 2]. Mutations in the CEBPA gene can occur across the whole coding region and have been described in approximately $6-24 \%$ of all acute myeloid leukemia (AML) patients[3-5]. CEBPAmutated (CEBPA ${ }^{\text {mut }}$ ) patients can be subdivided into two different subgroups: (1) AML carrying one mutation on one allele (singlemutated CEBPA, CEBPA ${ }^{\mathrm{sm}}$ ) and (2) AML with two CEBPA mutations (double-mutated CEBPA, CEBPA ${ }^{\mathrm{dm}}$ ), typically showing an Nterminal mutation and a basic leucine zipper gene mutation. However, only patients who harbor CEBPA double mutations are associated with favorable outcomes[6, 7]. In the 2016 World Health Organization (WHO) classification of leukemia, AML with CEBPA $^{\mathrm{dm}}$ was included as a distinctive disease entity due to its unique biological and clinical profiles[8].

With the application of next-generation sequencing (NGS), > 85\% of CEBPA ${ }^{\text {mut }}$ AML patients were observed to have other known concurrent mutations, such as WT1, CSF3R, and GATA2 $[9,10]$. However, studies have shown conflicting data on the impact of CEBPA mutations on prognosis when identified with other concomitant mutations[9-11]. These findings suggest that it is necessary to further explore co-mutations in the context of CEBPA mutations in AML.

Recently, mutations in the Wilms tumor 1 (WT1) gene have been observed in adult and childhood leukemia, commonly in AML[12, 13]. In several studies investigating more than 1000 AML samples total, mutations in WT1 were found in $2 \%-8.3 \%$ of the patients[12-15]. However, in AML patients with CEBPA ${ }^{\mathrm{dm}}$, the frequency of WT1 mutations increased to $13.6 \%-18.52 \%[10,14]$. To the best of our knowledge, few data are available regarding the impact of WT1 mutations on CEBPA ${ }^{\text {mut }}$ AML. We report here the frequency and types of WT1 mutations and their association with clinical features and outcomes in a cohort of 220 CEBPA ${ }^{\text {mut }}$ AML patients.

\section{Patients And Methods}

\section{Patients}


A total of 220 selected de novo AML patients with CEBPA mutations from five medical institutions of hematology (Affiliated Changzhou Second Hospital of Nanjing Medical University, Affiliated Hospital of Jiangnan University, Wuxi No. 2 People's Hospital, The Third Affiliated Hospital of Soochow University, and The First Affiliated Hospital of Soochow University) between August 2014 and November 2020 were analyzed. Of the patients, 114 were male and 106 were female. The median age was 39 years (range: 18-88 years), with 190 patients being $<60$ years and 30 patients being $\geq 60$ years. According to the French-American-British (FAB) classification, 14, 96, 33, 62, 5, and 10 patients were diagnosed with M1, M2, M4, M5, M6 and undetermined types, respectively. All patients gave their written informed consent for genetic analysis and for the use of the laboratory results for scientific studies. The study was approved by the research ethics board of each participating hospital and adhered to the tenets of the Declaration of Helsinki.

\section{Cytomorphology, cytogenetics and immunophenotyping}

Cytomorphologic assessment was performed by May-Grünwald-Giemsa staining, myeloperoxidase (MPO) reaction, and nonspecific esterase (NSE) staining using alpha-naphthyl-acetate following the FAB and WHO classifications. Karyotyping by the $\mathrm{G}$ or R-banding method was performed following standard methods on bone marrow (BM) cells after short culture. When possible, at least 20 metaphases were analyzed for each case. Immunophenotyping was performed as previously described[16].

\section{Mutation screening}

BM smear or peripheral blood (PB) samples at the time of initial diagnosis were collected. A sensitive next-generation amplicon deep-sequencing assay was used with an Illumina next-generation sequencer. A high depth of coverage (1000x) was obtained for 112 genes, including the whole coding regions that are known to be frequently mutated in hematologic malignancies. Altered DNA sequences were deemed mutations or variants in IGV software analysis and were identified by the COSMIC database and needed to exclude dbSNPs. Mutations in NPM1, FLT3, and CEBPA were identified by polymerase chain reaction (PCR) followed by direct Sanger sequencing, as previously described[17-19]. The mutations of the 112 genes analyzed in this study are listed in supplemental table 1 (Table S1).

\section{Fusion gene and WT1 mRNA expression}

Forty-one fusion genes were detected by multiple reverse transcription PCR amplification, which was performed as 8 parallel multiplex reactions on a 7500 Real-time PCR System (Applied Biosystems). We evaluated WT1 mRNA expression in BM or PB using a WT1 mRNA assay kit (Otsuka Pharmaceutical, Tokyo, Japan), as previously reported[20].

\section{Treatment and outcome}

Of the $220 \mathrm{AML}$ patients with CEBPA mutations, 161 patients received a standard chemotherapy regimen (DA/IA, daunorubicin/idarubicin $60 / 10 \mathrm{mg} / \mathrm{m}^{2} \mathrm{~d} 1-3$, cytarabine $100 \mathrm{mg} / \mathrm{m}^{2} \mathrm{~d} 1-7$ ) for induction therapy, 31 patients were prescribed a reduced intensity DA/IA (daunorubicin/idarubicin 45/6-8 mg/m² $\mathrm{d} 1-3$, cytarabine $100 \mathrm{mg} / \mathrm{m}^{2} \mathrm{~d} 1-7$ ) regimen, 13 patients received an HAD regimen (homoharringtonine $2 \mathrm{mg} / \mathrm{m}^{2} \mathrm{~d} 1-7$, cytarabine $100 \mathrm{mg} / \mathrm{m}^{2} \mathrm{~d} 1-7$, daunorubicin $40 \mathrm{mg} / \mathrm{m}^{2} \mathrm{~d} 1-3$ ), 14 patients received decitabine-based induction due to older age or underlying comorbidity, and 1 patient discontinued chemotherapy because of severe infection and respiratory failure.

Complete remission (CR) was observed in patients after one course of induction chemotherapy. Relapse-free survival (RFS) was defined as the time from diagnosis to relapse or the last follow-up in CR. Event-free survival (EFS) was calculated as the time interval from the date of diagnosis to the date of first evidence of an event, which included relapse, progression, recurrence, change, and death. Overall survival (OS) was defined as the time from diagnosis to death or the last follow-up.

\section{Statistical analysis}

Student's t-test was used to analyze continuous factors with a normal distribution, and the Mann-Whitney U test was used for comparisons of data that failed the normality test between different groups. Analysis of categorical variables between different cohorts was performed by the chi-square test and Fisher's exact test. Survival curves were calculated for RFS, OS and EFS based on the Kaplan-Meier method and compared using the two-sided log rank test. Patients who had undergone allogeneic bone 
marrow transplantation were censored at the time of transplantation. SPSS software version 20.0 (SPSS version 20.0; SPSS Institute, Chicago, IL, USA) was used for statistical analysis, and the results were considered significant at $P<0.05$.

\section{Results}

\section{Patients' characteristics}

A total of 99 patients (45.0\%) harboring single CEBPA mutations and 121 patients $(55.0 \%)$ with biallelic double mutations were identified. Compared with CEBPA ${ }^{\mathrm{sm}}$ AML patients, CEBPA ${ }^{\mathrm{dm}}$ AML patients had lower platelet counts (median: $29 \times 10^{9} / \mathrm{L} v s$. $43 \times 10^{9} / \mathrm{L}, P=0.002$ ), higher hemoglobin levels (median: $93 \mathrm{~g} / \mathrm{L} v s .82 \mathrm{~g} / \mathrm{L}, P=0.0162$ ), and lower WT1 expression levels (median: $12.385 \%$ vs. $42.35 \%, P=0.000)$. A total of $52.07 \%(63 / 121)$ of CEBPA ${ }^{\mathrm{dm}}$ AML patients presented with the FAB-M2 subtype, which occurred at a distinctively higher rate than that in CEBPA ${ }^{\text {sm }}$ patients $(52.07 \%$ vs. $33.33 \%, P=0.0053)$. AML patients with CEBPA ${ }^{\text {sm }}$ were more likely to show the M5 subtype than those with CEBPA ${ }^{\mathrm{dm}}(41.4 \%$ vs. $17.35 \%, P=0.0001)$. No significant differences were identified regarding sex, age, white blood cell (WBC) count or cytogenetic karyotype between the CEBPA ${ }^{\mathrm{sm}}$ and CEBPA ${ }^{\mathrm{dm}}$ AML groups. Table 1 lists different categories of clinical information of the enrolled patients.

\section{Frequency and types of WT1 mutations}

Overall, 30 WT1 mutations were detected in 29 of the 220 patients $(13.18 \%)$ screened. WT1 mutations occurred significantly more frequently in CEBPA ${ }^{d m}$ AML $(21 / 121,17.36 \%)$ than in $\operatorname{CEBPA}^{\mathrm{sm}}(8 / 99,8.08 \%)$ AML patients $(P=0.043)$. Mutations clustered overwhelmingly in exon 7 (16 mutations in 15 patients), but they were also detected in exon $8(n=6)$ and exon $9(n=8)$. One patient presented with two kinds of mutations (no. 29). The amino acid changes and mutation information of the WT1 gene are shown in Fig. 1 and Table 2.

\section{Association of WT1 mutations with clinical characteristics}

WT1-mutated (WT1 ${ }^{\text {mut }}$ ) patients had lower platelet counts (median: $25 \times 10^{9} / \mathrm{L} v s .38 \times 10^{9} / \mathrm{L}, P=0.0481$ ), higher WT1 expression levels (median: $29.72 \%$ vs. $16.81 \%, P=0.0371$ ), and a negative trend for the M5 subtype (median: $17.24 \%$ vs. $29.84 \%, P=0.07$ ) compared to WT wild-type (WT ${ }^{\mathrm{wt}}$ ) patients. No significant differences were identified regarding sex, age, WBC count, hemoglobin, or cytogenetic karyotype between the WT1 ${ }^{\text {mut }}$ and WT $1^{\text {wt }}$ groups. The clinical characteristics are shown in table 1.

\section{Companion gene mutations}

Based on NGS, we detected 59 mutated genes by screening the 112-gene panel. In addition to WT1, 33 of the 59 genes could be categorized as signaling pathways, epigenetic regulators, transcription factors, spliceosomes, tumor suppressors, chromatin modifiers, and cohesin. A total of $94.54 \%$ (208/220) of CEBPA mutations were accompanied by additional mutations and frequently co-occurred with FLT3-ITD ( $n=39,17.73 \%)$, NRAS ( $n=34,15.45 \%)$, GATA2 ( $n=34,15.45 \%)$, RUNX1 ( $n=30,13.64 \%)$, WT1 $(n=29,13.18 \%)$, FAT1 $(n=29,13.18 \%)$, TET2 $(n=23,10.45 \%)$, CSF3R $(n=20,9.09 \%)$, KIT $(n=21,9.54 \%)$ and NOTCH2 $(n=18,8.18 \%)$ Other genes had a mutation prevalence of $<8 \%$. Functionally, mutations involved in signaling pathways $(n=123,55.91 \%)$ and transcription factors $(n=47,21.36 \%)$ were found frequently in CEBPA ${ }^{\text {mut }}$ AML patients.

Among WT1-mutated patients, the most common co-mutation was FLT3-ITD ( $n=7,24.14 \%)$, followed by NRAS ( $n=5,17.24 \%)$, CSF3R $(n=4,13.79 \%)$, GATA2 $(n=4,13.79 \%)$, KIT $(n=4,13.79 \%)$, and FAT1 $(n=3,10.34 \%)$. Fewer common co-mutations ( $2 \%-$ $10.25 \%$ ) were found in NPM1, SF3B1, NOTCH1/2, PTPN11, JAK2 and others. The most frequent functional pathway was signaling pathways in as many as $62.07 \%$ of cases. Notably, no mutations in TET2, IDH1/2, or DNMT3A were identified in WT ${ }^{\text {mut }}$ patients. The concomitant mutations in epigenetic regulators were inversely correlated with WT1 mutations $(P=0.003)$. There were no differences in the incidences of FLT3-ITD, NRAS, CSF3R, GATA2, PTPN11, SF3B1 and NOTCH1/2 between WT ${ }^{\text {mut }}$ and WT ${ }^{\text {wt }}$ patients. The difference in the mutational spectrum between WT-mutated and WT1 wild-type AML is shown in table 3 . The frequencies of the detected gene mutations and gene functional groups are shown in Figure 2.

\section{Prognostic impact of WT1mutations in the context of CEBPA double mutations}


Of 121 patients with CEBPA double mutations, 111 patients were evaluable for complete remission (CR) after the first induction therapy. No differences were observed in the CR rate between patients with or without WT1 mutations in CEBPA ${ }^{\mathrm{dm}}$ AML (89.5\% vs. $91.3 \%, P=1.000$ ). The median follow up was 30.5 (range $0.5-60.6)$ months. We next investigated the impact of WT1 mutations on survival. WT1-mutated patients had significantly inferior RFS, EFS, and OS than WT1 wild-type patients (3-year RFS rate, 40\% vs. $70 \%$, median, 8.9 months vs. NR, $P=0.002$; 3-year EFS rate, 36\% vs. 65\%, median, 9.9 months vs. NR, $P=0.004 ; 3$-year OS rate, $48 \%$ vs. $72 \%, 20.4$ months vs. NR, $P=0.010$ ) (Fig. 3a/b/c and Table 4).

\section{Discussion}

The WT1 gene, located on chromosome 11p13, encodes a zinc-finger protein that exists in multiple isoforms and functions as a transcription factor involved in cellular growth, proliferation and differentiation and may therefore act as an oncogene[21-23]. The WT1 gene was initially identified as a tumor suppressor gene linked with nephroblastoma[24, 25]. Over the past years, numerous studies have highlighted that WT1 is aberrantly expressed or mutated in hematopoietic malignancies, including acute leukemia $(A L)$, myelodysplastic syndrome (MDS) and chronic myelogenous leukemia (CML)[26-30]. However, few data are available regarding the frequency of WT1 mutations and their impact on CEBPA ${ }^{\text {mut }}$ AML.

Recently, WT1 zinc-finger mutations (exons 7-10) were detected in $8.3 \%$ of pediatric AML patients[12], $10.6 \%$ of AML patients with a normal karyotype (NK-AML) [32], and 13.6\% of biallelic CEBPA ${ }^{\text {mut }}$ AML patients[14]. We revealed that WT1 mutations occurred in $17.36 \%$ of CEBPA ${ }^{\mathrm{dm}}$ AML patients in exons 7,8 and 9 . This percentage of cases is slightly higher than that reported in CEBPA ${ }^{\mathrm{dm}}$ AML by Krauth MT et al. (13.16\%) [14]. The difference could be explained by the fact that in the present study, we analyzed WT1 mutations in all exons, while in the study by Krauth MT et al., only mutations in exons 7 and 9 were analyzed. Similar to the findings of Fasan A et al.[3], we also found that WT1 was significantly more frequently mutated in CEBPA ${ }^{\mathrm{dm}}$ patients than in CEBPA $^{\text {sm }}$ patients, suggesting that the implication of the WT1 mutation may differ in CEBPA ${ }^{\mathrm{dm}}$ and CEBPA ${ }^{\mathrm{sm}}$ AML.

Zidan et al.[31] observed that WT1 mutations were significantly associated with younger age, and no associations were detected between the presence of WT1 mutations and sex, hemoglobin concentration, platelet count, and FAB subtype at diagnosis in NKAML. Haider I and colleagues[13] showed that there were also no statistically significant differences in clinical parameters, such as hemoglobin level, WBC count and platelet count, between WT1 ${ }^{\text {mut }}$ and WT1 ${ }^{\text {wt }}$ patients. Krauth MT et al.[14] found that WT1 mutations were more frequent in females than in males and in patients $<60$ years than in patients $\geq 60$ years $(95 / 1,449 ; 6.6 \%$ vs. $80 / 1,708 ; 4.7 \% ; P=0.014$ ). The median age of patients with WT1 mutations was lower (55.4 years compared to 67.5 years for WT1 wild-type) in AML; when the study population was limited to the subgroup with CEBPA ${ }^{\mathrm{dm}} \mathrm{AML}, 16.1 \%$ of WT1 ${ }^{\text {mut }}$ patients were $<60$ years old and $11.1 \%$ of WT $1^{\text {mut }}$ patients were $\geq 60$ years old $(P=0.317)$. In the present study, there was no significant difference between $\mathrm{WT}^{\text {mut }}$ and $\mathrm{WT} 1^{\mathrm{wt}} \mathrm{AML}$ patients with regard to age, sex, WBC count, and $\mathrm{Hb}$. However, WT $1^{\text {mut }}$ patients showed a significantly lower platelet count and a trend toward a negative association with FAB-M5 compared to WT ${ }^{\mathrm{Wt}}$ patients. The reason for these differences with the above studies might be due to the age distribution of the cohort, the patient enrollment criteria, the small sample size, the regions, or the research background.

Becker $\mathrm{H}$ et al. found that in WT1-mutated patients, FLT3-ITD more frequently occurred in older patients with primary NK-AML[33]. Krauth MT and colleagues found that WT1 mutations were rarely concomitant with DNMT3A $(4.4 \%, P=0.014)$, ASXL1 $(1.7 \%, P=$ 0.001), IDH2R140 (1.7\%, $P=0.001)$ and IDH1R132 (0.9\%, $P=0.001)$ mutations[14]. In this study, concomitant mutations in epigenetic regulators were inversely correlated with WT1 mutations $(P=0.003)$. This observation suggests that WT1 mutations are anticorrelated with epigenetic regulator mutations in CEBPA ${ }^{\text {mut }}$ AML. Rampal $R$ et al. observed that WT1-mutant AML patients had reduced 5-hydroxymethylcytosine (5hmC) levels, similar to TET2/IDH1/IDH2 mutant AML patients[34]. Wang Yiping et al. showed that WT1 physically interacts with and recruits TET2 to its target genes to activate their expression. The interaction between WT1 and TET2 is disrupted by multiple AML-derived TET2 mutations[35]. These findings, including our results, suggest that mutations in WT1 and TET2 may have interactive effects. Further studies are needed to reveal the interaction mechanism between WT1 and epigenetic regulators in the context of CEBPA mutations.

WT1 overexpression remains a valuable molecular marker in AML for predicting the survival of patients without any leukemiaspecific mutation, especially if NGS is not routinely available[26, 27]. Nagasaki $\mathrm{J}$ et al. showed that OS and progression-free 
survival (PFS) were significantly worse in elderly MDS patients with higher levels of WT1 at diagnosis[36]. Lyu Xiaodong et al. showed that WT1 overexpression by itself is an independent and negative indicator for predicting the CR rate, DFS and OS of NKAML patients[26]. Our data revealed that WT $1^{\text {mut }}$ patients showed significantly higher levels of WT1 expression than WT $1^{\text {wt }}$ patients, but further investigation is needed to explore the effect of WT1 expression on prognosis in the background of WT $^{\text {mut }} /$ CEBPA ${ }^{\text {mut }}$ AML.

There are still some controversies regarding the prognostic significance of WT1 mutations in patients with AML. Studies from Hou HA and Virappane P demonstrated that the WT1 mutation was associated with poor prognosis in NK-AML and nonselective AML patients[37, 38]. Tien Feng-Ming and colleagues found that WT1 ${ }^{\text {mut }}$ patients with CEBPA ${ }^{\mathrm{dm}}$ AML tended to have a lower CR rate, a higher relapse rate, and significantly shorter OS and disease-free survival (DFS) than those with wild-type WT1[39]. Krauth MT et al. indicated that WT1 ${ }^{\text {mut }}$ was associated with shorter EFS in NK-AML patients, but it had no impact on prognosis in subgroups with high WT1 ${ }^{\text {mut }}$ incidences (CEBPA ${ }^{\mathrm{dm}}$, PML-RARA)[14]. An analysis based on the TCGA database showed that the WT1-mutated group had lower rates of $C R$ but higher rates of minimal residual disease (MRD) after one and two courses of induction chemotherapy. In NK-AML pediatric patients, those with WT1 mutations had significantly worse OS and EFS in both univariate and multivariate survival analyses[40]. In our study, WT1 ${ }^{\text {mut }}$ patients had significantly inferior 3-year RFS, EFS and OS rates than WT $1^{\text {wt }}$ patients in the context of CEBPA double mutations. This result is consistent with that reported by Tien Feng-Ming et al. but different from that reported by Krauth MT et al. This inconsistency may be attributed to differences in the characteristics of the populations investigated, such as sex, karyotype, age, combination with other mutations such as FLT3-ITD, or different ethnicities.

In conclusion, this study confirms that WT1 mutations are frequently identified in CEBPA ${ }^{\text {mut }}$ AML patients, especially in CEBPA ${ }^{\mathrm{dm}}$ AML patients. Patients with WT1 mutations show distinct clinical features, a spectrum of co-mutations, and poor prognosis compared to WT1 wild-type patients.

\section{Declarations}

\section{Ethics approval and consent to participate}

All patients gave their written informed consent for genetic analysis and for the use of the laboratory results for scientific studies. The study was performed in accordance with the World Medical Association Declaration of Helsinki and Ethics Committee approval of Changzhou No.2 People's Hospital was obtained ([2015]KY004-1).

\section{Consent for publication}

Written informed consent for publication was obtained from all participants.

\section{Availability of data and materials}

The datasets used and/or analysed during the current study available from the corresponding author on reasonable request.

\section{Competing interests}

The authors declare no competing financial interests.

\section{Funding}

This work was partly supported by grants from Young Scientists Foundation of Changzhou No. 2 People's Hospital (N0.2019K002), Science and Technology Project of Changzhou Health Committee (NO.QN202035), Science and Technology Development Fund project of Nanjing Medical University(No. NMUB201), Major Science Project of Changzhou Health Commission(ZD202018).

\section{Authors' contributions}

TW, HYH, BW, WQ, PW and HYC had contributions in collecting clinical information of selected patients. ZW, BW, HYC and PW completed the entire experimental processes. TW and HYC completed all statistical analyses and constructed figures. TW wrote 
the manuscript. HYC and XZL were the main contributors in revising the manuscript. Other authors provided some advice on the manuscript. All authors read and approved the final manuscript.

\section{Acknowledgements}

The authors thank all the physicians who provided leukemia samples and clinical data.

\section{References}

1. Steffen Koschmieder, Balazs Halmos, Elena Levantini, Daniel G Tenen. Dysregulation of the C/EBPalpha differentiation pathway in human cancer. J Clin Oncol. 2009;27(4): 619-628.

2. Quintana-Bustamante O, Lan-Lan Smith S, Griessinger E, et al. Overexpression of wild-type or mutants forms of CEBPA alter normal human hematopoiesis. Leukemia. 2012;26(7): 1537-15

3. Fasan A, Haferlach C, Alpermann T, et al. The role of different genetic subtypes of CEBPA mutated AML. Leukemia. 2014;28(4): 794-803.

4. Ahmad F, Rajput S, Mandava S, Das BR. Molecular Evaluation of CEBPA Gene Mutation in Normal Karyotype Acute Myeloid Leukemia: A Comparison of Two Methods and Report of Novel CEBPA Mutations from Indian Acute Myeloid Leukemia Patients. Genet Test Mol Biomarkers. 2012;16(7): 707-715.

5. Ahn JY, Seo K, Weinberg O, BoyD SD, Arber DA. A Comparison of two methods for screening CEBPA mutations in patients with acute myeloid leukemia. J Mol Diagn. 2009;11(4): 319-323.

6. Pabst T, Eyholzer M, Fos J, Mueller BU. Heterogeneity within AML with CEBPA mutations; only CEBPA double mutations, but not single CEBPA mutations are associated with favourable prognosis. Br J Cancer. 2009;100(8): 1343-1346.

7. Taskesen $E$, Bullinger $L$, Corbacioglu $A$, et al. Prognostic impact, concurrent genetic mutations, and gene expression features of AML with CEBPA mutations in a cohort of 1182 cytogenetically normal AML patients: Further evidence for CEBPA double mutant AML as a distinctive disease entity. Blood. 2011;117(8): 2469-24

8. Arber DA, Orazi A, Hasserjian R, et al. The 2016 revision to the World Health Organization classification of myeloid neoplasms and acute leukemia. Blood. 2016;127(20): 2391-2405.

9. Zhang $Y$, Wang $F$, Chen $X$, et al. Companion gene mutations and their clinical significance in AML with double mutant Cancer Gene Ther. 2020;27(7-8): 599-606.

10. Su L, Tan Y, Lin H, et al. Mutational spectrum of acute myeloid leukemia patients with double CEBPA mutations based on next-generation sequencing and its prognostic significance. Oncotarget. 2018;9(38): 24970-249

11. Grossmann V, Haferlach C, Nadarajah N, et al. CEBPA double-mutated acute myeloid leukaemia harbours concomitant molecular mutations in $76.8 \%$ of cases with TET2 and GATA2 alterations impacting prognosis. Br J Haematol. 2013;161(5): 649-6

12. Ho PA, Zeng R, Alonzo TA, et al. Prevalence and prognostic implications of WT1 mutations in pediatric acute myeloid leukemia (AML): a report from the Children's Oncology Group. Blood. 2010;116(5): 702-7

13. Haider I, Kumar C, Jain G, et al. Hotspots mutational analysis of Wilms tumor 1 gene in acute myeloid leukaemia; prevalence and clinical correlation in North Indian population. Am J Blood Res. 2020;10(5): 179-189.

14. Krauth MT, Alpermann T, Bacher U, et al. WT1 mutations are secondary events in AML, show varying frequencies and impact on prognosis between genetic subgroups. Leukemia. 2015;29(3): 660-66

15. Luo S, Yu K, Yan QX, et al. Analysis of WT1 mutations, expression levels and single nucleotide polymorphism rs 16754 in de novo non-M3 acute myeloid leukemia. Leuk Lymphoma. 2014;55(2): 349-3

16. Kern W, Bacher U, Haferlach C, Schnittger S, Haferlach. The role of multiparameter flow cytometry for disease monitoring in AML. Best Pract Res Clin Haematol. 2010;23(3): 379-390.

17. Pabst T, Mueller BU, Zhang P, et al. Dominant-negative mutations of CEBPA, encoding CCAAT/enhancer binding protein-alpha (C/EBPalpha), in acute myeloid leukemia. Nat Genet. 2001;27(3): 263-270. 
18. Kumar D, Mehta A, Panigrahi MK, Nath S, Saikia KK. NPM1 Mutation Analysis in Acute Myeloid Leukemia: Comparison of Three Techniques -Sanger Sequencing, Pyrosequencing, and Real-Time Polymerase Chain Reaction. Turk J Haematol. 2018;35(1): 49-53.

19. Thiede C, Steudel C, Mohr B, et al. Analysis of FLT3-activating mutations in 979 patients with acute myelogenous leukemia: association with FAB subtypes and identification of subgroups with poor prognosis. Blood. 2002;99(12): 4326-4335.

20. Kitamura K, Nishiyama T, Ishiyama K, et al. Clinical usefulness of WT1 mRNA expression in bone marrow detected by a new WT1 mRNA assay kit for monitoring acute myeloid leukemia: a comparison with expression of WT1 mRNA in peripheral blood. Int J Hematol. 2016;103(1): 53-62.

21. Call KM, Glaser T, Ito CY, et al. Isolation and characterization of a zinc finger polypeptide gene at the human chromosome 11 Wilms' tumor locus. Cell. 1990;60(3): 509-520.

22. Yang L, Han Y, Suarez SF, Minden MD. A tumor suppressor and oncogene: the WT1 story. Leukemia. 2007;21(5): 868-876.

23. Yamagami T, Sugiyama H, Inoue K, et al. Growth inhibition of human leukemic cells by WT1 (Wilms tumor gene) antisense oligodeoxynucleotides: implications for the involvement of WT1 in leukemogenesis. Blood. 1996;87(7): 2878-2884.

24. Sugiyama H. Wilms' tumor gene WT1: its oncogenic function and clinical application. Int J Hemato 2001;73(2): $177-187$.

25. Huff V. Wilms' tumours: about tumour suppressor genes, an oncogene and a chameleon gene. Nat Rev Cancer. 2011;11(2): $111-121$.

26. Lyu X, Xin Y, Mi R, et al. Overexpression of Wilms Tumor 1 Gene as a Negative Prognostic Indicator in Acute Myeloid Leukemia. PLoS One. 2014;9(3):

27. Šálek C, Vydra J, Cerovská E, et al. WT1 Expression in Peripheral Blood at Diagnosis and During the Course of Early Consolidation Treatment Correlates With Survival in Patients With Intermediate and Poor-Risk Acute Myeloid Leukemia. Clin Lymphoma Myeloma Leuk. 2020;20(12): 998-1009.

28. Ahmad F, D'Souza W, Mandava S, Das BR. Molecular analysis of WT1 and KIT mutations in patients from an Indian population with de novo acute myeloid leukemia: determination of incidence, distribution patterns, and report of a novel KIT Leuk Lymphoma. 2011;52(5): 865-876.

29. Luo P, Jing W, Yi K, Wu S, Zhou F. Wilms' tumor 1 gene in hematopoietic malignancies: clinical implications and future directions. Leuk Lymphoma. 2020;61(9): 2059-20

30. Zhu H, Yang B, Liu J, Wu W, Lingc Y. Case Report of acute myeloid leukemia with "WT1, ATRX, CEBPA, CSMD1, IKZF1, and LRP1B mutation and translocation between chromosome 1 and 19" developing from Philadelphia-negative chronic myeloid leukemia after TKI therapy. Medicine (Baltimore). 2020;99(3):

31. Zidan MA, Kamal Shaaban HM, Elghannam DM. Prognostic impact of Wilms tumor gene mutations in Egyptian patients with acute myeloid leukemia with normal karyotype. Hematology. 2014;19(5): 267-2

32. Aref S, El Sharawy S, Sabry M, Azmy E, Raouf DA. Prognostic relevance of Wilms tumor 1 (WT1) gene Exon 7 mutations inpatient with cytogenetically normal acute myeloid leukemia. Indian J Hematol Blood Transfus. 2014;30(4): 226-2

33. Becker H, Marcucci G, Maharry K, Radmacher MD, Mrózek K. Mutations of the Wilms tumor 1 gene (WT1) in older patients with primary cytogenetically normal acute myeloid leukemia: a Cancer and Leukemia Group B study. Blood. 2010;116(5): 7887

34. Rampal R, Alkalin A, Madzo J, et al. DNA Hydroxymethylation Profiling Reveals that WT1 Mutations Result in Loss of TET2 Function in Acute Myeloid Leukemia. Cell Rep. 2014;9(5): 1841-18

35. Wang Y, Xiao M, Chen X, et al. WT1 Recruits TET2 to Regulate Its Target Gene Expression and Suppress Leukemia Cell Proliferation. Mol Cell. 2015;57(4): 662-673.

36. Nagasaki J, Aoyama Y, Hino M, et al. Wilms Tumor 1 (WT1) mRNA Expression Level at Diagnosis Is a Significant Prognostic Marker in Elderly Patients with Myelodysplastic Syndrome. Acta Haematol. 2017;137(1): 32-39.

37. Hou HA, Huang TC, Lin LI, et al. WT1 mutation in 470 adult patients with acute myeloid leukemia: stability during disease evolution and implication of its incorporation into a survival scoring system. Blood. 2010;115(25): 5222-5231.

38. Virappane P, Gale R, Hills R, et al. Mutation of the Wilms' tumor 1 gene is a poor prognostic factor associated with chemotherapy resistance in normal karyotype acute myeloid leukemia: the United Kingdom Medical Research Council Adult 
Leukaemia Working Party. J Clin Oncol. 2008;26(33): 5429-5435.

39. Tien FM, Hou HA, Tang JL, et al. Concomitant WT1 mutations predict poor prognosis in acute myeloid leukemia patients with double mutant Haematologica. 2018;103(11): 510-513.

40. Xu J, Zhang Y, Hu J, Ren Y, Wang H. Clinical features and prognosis of normal karyotype acute myeloid leukemia pediatric patients with WT1 mutations: an analysis based on TCGA database. Hematology. 2020;25(1): 79-84.

\section{Tables}

Table 1. Pretreatment clinical characteristics according to CEBPA and WT1 mutation status 


\begin{tabular}{|c|c|c|c|c|c|c|c|}
\hline Variable & $\begin{array}{l}\text { Total } \\
(n=220)\end{array}$ & CEBPA $^{\text {sm }}(n=99)$ & CEBPA $^{\mathrm{dm}}(\mathrm{n}=121)$ & $P 1$ & $W^{\text {mut }}(\mathbf{n}=29)$ & WT1 ${ }^{w t}(n=191)$ & $P 2$ \\
\hline \multicolumn{8}{|l|}{ Gender } \\
\hline Male, n (\%) & $\begin{array}{l}114( \\
51.8 \%)\end{array}$ & $46(46.5 \%)$ & $68(56.2 \%)$ & \multirow[t]{2}{*}{0.1506} & $12(41.38 \%)$ & $102(53.4 \%)$ & \multirow[t]{2}{*}{0.2273} \\
\hline Female, n (\%) & $\begin{array}{l}106( \\
48.18 \%)\end{array}$ & $53(53.5 \%)$ & $53(43.8 \%)$ & & $17(58.62 \%)$ & $89(46.6 \%)$ & \\
\hline \multicolumn{8}{|l|}{ Age (year) } \\
\hline Median (range) & $\begin{array}{l}39(18- \\
88)\end{array}$ & $38(18-76)$ & $40(18-88)$ & 0.2371 & $43(18-66)$ & 39 (18-78) & 0.7165 \\
\hline \multicolumn{8}{|l|}{ WBC (×109/L) } \\
\hline Median (range) & $\begin{array}{l}16.21 \\
(0.61- \\
287.6)\end{array}$ & $12(0.61-195.7)$ & $18.67(1.7-287.6)$ & 0.3663 & $\begin{array}{l}18.67(0.8- \\
195.7)\end{array}$ & $\begin{array}{l}15.7(0.61- \\
287.6)\end{array}$ & 0.6242 \\
\hline \multicolumn{8}{|l|}{$\mathrm{Hb}(\mathrm{g} / \mathrm{L})$} \\
\hline Median (range) & $\begin{array}{l}87.5(35- \\
158)\end{array}$ & $82(35-151)$ & $93(55-158)$ & 0.0162 & $91(35-158)$ & $87(49-147)$ & 0.5934 \\
\hline \multicolumn{8}{|l|}{ PLT $\left(\times 10^{9} / \mathrm{L}\right)$} \\
\hline Median (range) & $\begin{array}{l}36.5(5- \\
389)\end{array}$ & $43(11-389)$ & $29(5-322)$ & 0.002 & $25(5-129)$ & 38 (7-389) & 0.0481 \\
\hline \multicolumn{8}{|l|}{$\begin{array}{l}\text { Cytogenetic } \\
\text { karyotype }\end{array}$} \\
\hline normal, n (\%) & $\begin{array}{l}167 \\
(76.6 \%)\end{array}$ & 73 (74.5\%) & $94(78.3 \%)$ & & $22(75.9 \%)$ & $145(76.72 \%)$ & \\
\hline abnormal, n (\%) & $\begin{array}{l}51 \\
(23.4 \%)\end{array}$ & $25(25.5 \%)$ & $26(21.7 \%)$ & 0.5049 & $7(24.1 \%)$ & $44(23.28 \%)$ & 0.9192 \\
\hline NA & 2 & 1 & 1 & & 0 & 2 & \\
\hline \multicolumn{8}{|l|}{$\begin{array}{l}\text { WT1 expression } \\
(\%)\end{array}$} \\
\hline Median (Range) & $\begin{array}{l}18.74 \\
(0.01- \\
317)\end{array}$ & $\begin{array}{l}42.35(0.01- \\
213.07)\end{array}$ & $\begin{array}{l}12.385(0.21- \\
317)\end{array}$ & 0.000 & $\begin{array}{l}29.72(8.44- \\
294)\end{array}$ & $\begin{array}{l}16.81(0.01- \\
317)\end{array}$ & 0.0371 \\
\hline NA & 59 & & & & 8 & 51 & \\
\hline \multicolumn{8}{|l|}{ FAB classification } \\
\hline M1, n(\%) & $\begin{array}{l}14 \\
(6.36 \%)\end{array}$ & $6(6.06 \%)$ & $8(6.61 \%)$ & 0.8768 & $1(3.44 \%)$ & $13(6.8 \%)$ & 0.3 \\
\hline M2, n(\%) & $\begin{array}{l}96 \\
(43.63 \%)\end{array}$ & $33(33.33 \%)$ & $63(52.07 \%)$ & 0.0053 & $16(55.17 \%)$ & $80(41.88 \%)$ & 0.1788 \\
\hline M4, n(\%) & $\begin{array}{l}33 \\
(15.0 \%)\end{array}$ & $11(11.11 \%)$ & $22(18.2 \%)$ & 0.1440 & $5(17.24 \%)$ & $28(14.66 \%)$ & 0.2 \\
\hline M5, n(\%) & $\begin{array}{l}62 \\
(28.18 \%)\end{array}$ & $41(41.4 \%)$ & $21(17.35 \%)$ & 0.0001 & $5(17.24 \%)$ & $57(29.84 \%)$ & 0.07 \\
\hline M6, n(\%) & $\begin{array}{l}5 \\
(2.27 \%)\end{array}$ & $3(3.03 \%)$ & $2(1.65 \%)$ & 0.28 & 0 & $5(2.62 \%)$ & 1 \\
\hline Undetermined,n(\%) & $\begin{array}{l}10 \\
(4.54 \%)\end{array}$ & $5(5.05 \%)$ & $5(4.13 \%)$ & 0.24 & $2(6.89 \%)$ & $8(4.19 \%)$ & 0.26 \\
\hline
\end{tabular}


Abbreviations: $P, p$-value, $P 1$, CEBPA ${ }^{\text {sm }}$ AML group vs.CEBPA ${ }^{\mathrm{dm}} \mathrm{AML}$ group; $P 2, \mathrm{WT} 1^{\text {mut }} \mathrm{AML}$ group $v s$. WT $1^{\text {wt }} \mathrm{AML}$ group; $\mathrm{n}$, number of patients; $A M L$,acute myeloid leukemia; $F$, female; $M$, male; WBC, white blood cell count, HB, hemoglobin; PLT, platelet; NA, not available.

Table 20WT1 mutations information in 29 patients with CEBPA ${ }^{\text {mut }}$ AML 


\begin{tabular}{|c|c|c|c|c|c|c|c|c|}
\hline $\begin{array}{l}\text { Case No. } \\
\text { /Sex/Age(y) }\end{array}$ & $\begin{array}{l}\text { WBC } \\
\left(\times 10^{9} / \mathrm{L}\right)\end{array}$ & $\begin{array}{l}H B \\
(g / L)\end{array}$ & $\begin{array}{l}\text { PLT } \\
\left(\times 10^{9} / \mathrm{L}\right)\end{array}$ & $\begin{array}{l}\text { FAB } \\
\text { subtype }\end{array}$ & Karyotype & $\begin{array}{l}\text { WT1mut } \\
\text { a.a. } \\
\text { change }\end{array}$ & $\begin{array}{l}\text { Multiple } \\
\text { RT-PCR }\end{array}$ & $\begin{array}{l}\text { WT1 } \\
\text { expression(\%) }\end{array}$ \\
\hline 1/Male/54 & 10.27 & 87 & 25 & M2 & $47, X Y,+22$ & S435X & $(-)$ & 76.57 \\
\hline 2/Male/18 & 37 & 66 & 50 & M2 & $\begin{array}{l}47, X Y,+8, \operatorname{del}(9) \\
\text { (q13q22) }\end{array}$ & A382fs & $(-)$ & NA \\
\hline 3/Female/49 & 62.14 & 74 & 66 & M4 & $46, X X, \operatorname{der}(7)$ & R440fs & $(-)$ & 28.15 \\
\hline 4/Male/52 & 0.8 & 62 & 38 & M5 & $45, X Y,-7$ & R462P & $(-)$ & 294 \\
\hline 5/Female/23 & 18.9 & 102 & 44 & M2 & $45, X X,-8$ & A382fs & $(-)$ & 29.72 \\
\hline 6/Female/43 & 37 & 91 & 18 & Undetermined & $\begin{array}{l}46, X X, \operatorname{del}(9) \\
\text { (q13q22) }\end{array}$ & R370fs & $(-)$ & 78.03 \\
\hline 7/Female/66 & 17.4 & 63 & 5 & M2 & $\begin{array}{l}46, X X, \operatorname{del}(9) \\
\text { (q13q22) }\end{array}$ & A382fs & $(-)$ & 184.66 \\
\hline 8/Female/34 & 195.7 & 57 & 23 & M4 & $46, X X$ & R462L & $(-)$ & NA \\
\hline 9/Female/40 & 15.0 & 93 & 57 & M4 & $46, X X$ & S381X & $(-)$ & 10.66 \\
\hline 10/Male/54 & 46.9 & 98 & 9 & M4 & $46, X Y$ & S381X & $(-)$ & 12.82 \\
\hline 11/Male/20 & 4.0 & 61 & 27 & M1 & $46, X Y$ & S381X & $(-)$ & NA \\
\hline 12/Female/18 & 86.0 & 104 & 20 & M2 & $46, X X$ & R462P & $(-)$ & NA \\
\hline 13/Female/46 & 11.9 & 79 & 29 & M5 & $46, X X$ & K414fs & $(-)$ & 8.44 \\
\hline 14/Female/62 & 4.11 & 65 & 25 & Undetermined & $46, X X$ & $\mathrm{H} 465 \mathrm{Y}$ & $(-)$ & NA \\
\hline 15/Male/60 & 10.39 & 132 & 26 & M5 & $46, X Y$ & A382fs & $(-)$ & NA \\
\hline 16/Male/57 & 8.15 & 106 & 17 & M2 & $46, X Y$ & $\mathrm{R} 462 \mathrm{P}$ & $(-)$ & 67.22 \\
\hline 17/Male/66 & 27.88 & 119 & 16 & M2 & $46, X Y$ & S435X & $(-)$ & 47.44 \\
\hline 18/Female/30 & 90.13 & 105 & 24 & M5 & $46, X X$ & $\mathrm{R} 434 \mathrm{H}$ & $(-)$ & 27.89 \\
\hline 19/Male/62 & 8.96 & 103 & 27 & M2 & $46, X Y$ & S381X & $(-)$ & 16.7 \\
\hline 20/Female/19 & 50.31 & 63 & 23 & M2 & $46, X X$ & R380G & $(-)$ & 22.51 \\
\hline 21/Female/50 & 7.07 & 88 & 16 & M2 & $46, X X$ & A382fs & $(-)$ & 204.18 \\
\hline 22/Female/45 & 35 & 128 & 23 & M4 & $46, X X$ & V379fs & $(-)$ & 160.35 \\
\hline 23/Male/22 & 50.24 & 35 & 21 & M2 & $46, X Y$ & H465G & $(-)$ & 18.84 \\
\hline 24/Male/29 & 4.44 & 158 & 129 & M2 & $46, X Y$ & $\mathrm{H} 445 \mathrm{fs}$ & $(-)$ & 21.27 \\
\hline 25/Female/20 & 18.67 & 102 & 23 & M2 & $46, X X$ & R462L & $(-)$ & 82.69 \\
\hline 26/Male/19 & 76.79 & 91 & 38 & M5 & $46, X Y$ & R380fs & $(-)$ & 42.02 \\
\hline 27/Male/54 & 9.28 & 93 & 38 & M2 & $46, X Y$ & C423Y & $(-)$ & 17.03 \\
\hline 28/Female/30 & 2.09 & 85 & 54 & M2 & $46, X X$ & R462P & $(-)$ & NA \\
\hline 29/Female/30 & 79.74 & 63 & 53 & M2 & $46, X X$ & $\begin{array}{l}\text { R380G, } \\
\text { S381fs }\end{array}$ & $(-)$ & NA \\
\hline
\end{tabular}

Abbreviations:AML,acute myeloid leukemia (AML) ; F, female; M, male; WBC, white blood cell count, HB, hemoglobin; PLT, platelet; a.a., amino acid; 
$\mathrm{NA}$, not available; (+), positive; (-), negative $\mathbb{R} \mathrm{R}-\mathrm{PCR}$, reverse transcription polymerase chain reaction

Table 3. Concomitant gene abnormalities according to WT1 mutation status 


\begin{tabular}{|c|c|c|c|c|}
\hline Mutational genes & Total $(n=220)$ & WT1 ${ }^{\text {mut }}$ AML $(n=29)$ & WT $1^{\text {wt }}$ AML $(n=191)$ & $P$ \\
\hline Signaling pathways, n (\%) & $123(123 / 220,55.91 \%)$ & $17(18 / 29,62.07 \%)$ & $106(106 / 191,55.59 \%)$ & 0.506 \\
\hline c-KIT, n (\%) & $21(21 / 220,9.54 \%)$ & $4(4 / 29,13.79 \%)$ & $17(17 / 191,8.9 \%)$ & 0.493 \\
\hline NRAS, n (\%) & $34(34 / 220,15.45 \%)$ & $5(5 / 29,17.24 \%)$ & $29(29 / 191,15.18 \%)$ & 0.784 \\
\hline KRAS, n (\%) & $7(7 / 220,3.18 \%)$ & 0 & $7(7 / 191,3.66 \%)$ & 0.598 \\
\hline FLT3-ITD, n (\%) & $39(39 / 220,17.73 \%)$ & $7(7 / 29,24.14 \%)$ & $32(32 / 191,16.75 \%)$ & 0.332 \\
\hline CSF3R, n (\%) & $20(20 / 220,9.09 \%)$ & $4(4 / 29,13.79 \%)$ & $16(16 / 191,8.38 \%)$ & 0.311 \\
\hline RELN, n (\%) & $13(13 / 220,5.91 \%)$ & $2(2 / 29,6.89 \%)$ & $11(11 / 191,5.75 \%)$ & 0.268 \\
\hline NOTCH1, n (\%) & $11(11 / 220,5.0 \%)$ & $1(1 / 29,3.45 \%)$ & $10(10 / 191,5.23 \%)$ & 1 \\
\hline NOTCH2, n (\%) & $18(18 / 220,8.18 \%)$ & $2(2 / 29,6.89 \%)$ & $16(16 / 191,8.38 \%)$ & 1 \\
\hline JAK2, n (\%) & $4(4 / 220,1.82 \%)$ & $1(1 / 29,3.45 \%)$ & $3(3 / 191,1.57 \%)$ & 0.434 \\
\hline SH2B3, n (\%) & $8(8 / 220,3.64 \%)$ & $1(1 / 29,3.45 \%)$ & $7(7 / 191,3.66 \%)$ & 1 \\
\hline PTPN11, n (\%) & $11(11 / 220,5.0 \%)$ & $2(2 / 29,6.89 \%)$ & $9(9 / 191,4.71 \%)$ & 1 \\
\hline Epigenetic regulators, $\mathrm{n}(\%)$ & $41(41 / 220,18.64 \%)$ & 0 & $41(41 / 191,21.46 \%)$ & 0.003 \\
\hline TET2, n (\%) & $23(23 / 220,10.45 \%)$ & 0 & $23(23 / 191,12.04 \%)$ & 0.05 \\
\hline IDH1, n (\%) & $9(9 / 220,4.09 \%)$ & 0 & $9(9 / 191,4.71 \%)$ & 0.610 \\
\hline IDH2, n (\%) & $3(3 / 220,1.36 \%)$ & 0 & $3(3 / 191,1.57 \%)$ & 1 \\
\hline DNMT3A, n (\%) & $14(14 / 220,6.36 \%)$ & 0 & $14(14 / 191,7.33 \%)$ & 0.225 \\
\hline Transcription factors, n (\%) & $47 *(47 / 220,21.36 \%)$ & $4 *(4 / 29,13.79 \%)$ & $43 *(43 / 191,22.5 \%)$ & 0.286 \\
\hline ETV6, n (\%) & $1(1 / 220,0.45 \%)$ & 0 & $1(1 / 191,0.52 \%)$ & 1 \\
\hline RUNX1, n (\%) & $30(30 / 220,13.64 \%)$ & 0 & $6(6 / 191,3.14 \%)$ & 1 \\
\hline GATA2, n (\%) & $34(34 / 220,15.45 \%)$ & $4(4 / 29,13.79 \%)$ & $30(30 / 191,15.71 \%)$ & 1 \\
\hline SETBP1, n (\%) & $9(9 / 220,4.09 \%)$ & 0 & $9(9 / 191,4.71 \%)$ & 0.607 \\
\hline CEBPA $^{\mathrm{dm}}, \mathrm{n}(\%)$ & $121(121 / 220,55 \%)$ & $21(21 / 29,72.4 \%)$ & $100(100 / 191,52.36 \%)$ & \multirow[t]{2}{*}{0.043} \\
\hline CEBPA $^{\mathrm{Sm}}, \mathrm{n}(\%)$ & $99(99 / 220,45.0 \%)$ & $8(8 / 29,27.58 \%)$ & $91(91 / 191,47.64 \%)$ & \\
\hline Spliceosomes, n (\%) & $9(9 / 220,4.09 \%)$ & $1(1 / 29,3.45 \%)$ & $8(8 / 191,4.19 \%)$ & 1 \\
\hline SRSF2, n (\%) & $1(1 / 220,0.45 \%)$ & 0 & $1(1 / 191,0.52 \%)$ & 1 \\
\hline SF3B1, n (\%) & $6(6 / 220,2.73 \%)$ & $1(1 / 29,3.45 \%)$ & $5(5 / 191,2.62 \%)$ & 0.576 \\
\hline ZRSR2, n (\%) & $2(2 / 220,0.90 \%)$ & 0 & $2(2 / 191,1.05 \%)$ & 1 \\
\hline Tumour suppressors, n (\%) & $38(38 / 220,17.27 \%)$ & $3(3 / 29,10.34 \%)$ & 35 (35/191, 18.32\%) & 0.429 \\
\hline TP53, n (\%) & $9(9 / 220,4.09 \%)$ & 0 & $9(9 / 191,4.71 \%)$ & 0.610 \\
\hline FAT1, n (\%) & $29(29 / 220,13.18 \%)$ & $3(3 / 29,10.34 \%)$ & $26(26 / 191,13.61 \%)$ & 0.775 \\
\hline Cohesin, n (\%) & $(7 / 191,3.66 \%)$ & 0 & 7 (7/191, 3.66\%) & 0.598 \\
\hline RAD21, n (\%) & $5(5 / 220,2.27 \%)$ & 0 & $5(5 / 191,2.62 \%)$ & 1 \\
\hline SMC1A, n (\%) & $1(1 / 220,0.45 \%)$ & 0 & $1(1 / 191,0.52 \%)$ & 1 \\
\hline SMC3, n (\%) & $1(1 / 220,0.45 \%)$ & 0 & $1(1 / 191,0.52 \%)$ & 1 \\
\hline
\end{tabular}




\begin{tabular}{|lllll|}
\hline Chromatin modifiers, $\mathbf{n}(\%)$ & $24(24 / 220,10.91 \%)$ & $1(1 / 29,3.45 \%)$ & $23(23 / 191,12.04 \%)$ & 0.215 \\
\hline ASXL1, n (\%) & $10(10 / 220,4.55 \%)$ & 0 & $10(10 / 191,5.23 \%)$ & 0.366 \\
\hline EZH2, n (\%) & $8(8 / 220,3.64 \%)$ & $1(1 / 29,3.45 \%)$ & $8(8 / 191,4.19 \%)$ & 1 \\
\hline BCOR, n (\%) & $5(5 / 220,2.27 \%)$ & 0 & $1(1 / 191,0.52 \%)$ & 1 \\
\hline BCORL1, n (\%) & $4(4 / 220,1.82 \%)$ & 0 & $4(4 / 191,2.09 \%)$ & 1 \\
\hline
\end{tabular}

Abbreviations: $P, p$-value, WT1 ${ }^{\text {mut }}$ AML group $v s$. WT $1^{\text {wt }}$ AML group; $\mathrm{n}$, number of patients; AML, acute myeloid leukemia; *The WT1 mutation was excluded.

Table 4. Univariable outcome analyses according to WT1 mutation status

\begin{tabular}{|c|c|c|c|c|}
\hline Clinical endpoint & $\begin{array}{l}\text { Total } \\
(n=121)\end{array}$ & $\begin{array}{l}\mathrm{WT} 1^{\mathrm{mut}} / \mathrm{CEBPA}^{\mathrm{dm}} \\
(\mathrm{n}=21)\end{array}$ & $\begin{array}{l}\text { WT1 }{ }^{w t} / \text { CEBPA }^{d m} \\
(n=100)\end{array}$ & $P$ \\
\hline $\mathrm{CR}$ rate, $\%$ & $101 / 111,91.0 \%$ & $17 / 19,89.5 \%$ & $84 / 92,91.3 \%$ & 1.000 \\
\hline missing, $\mathrm{n}$ & 10 & $2^{*}$ & 8 & \\
\hline RFS & & & & 0.002 \\
\hline Median, mo $(95 \% \mathrm{Cl})$ & NR & $8.9(0-21.7)$ & NR & \\
\hline 3-year DFS, \% (SE) & $66(5)$ & $40(14)$ & $70(6)$ & \\
\hline missing, $\mathrm{n}$ & 19 & 4 & 15 & \\
\hline EFS & & 2 & 7 & 0.004 \\
\hline Median, mo $(95 \% \mathrm{Cl})$ & NR & $9.9(8.4-11.4)$ & NR & \\
\hline 3-year EFS, \% (SE) & $61(5)$ & $36(12)$ & $65(5)$ & \\
\hline missing, $\mathrm{n}$ & 9 & 2 & 7 & \\
\hline OS & & & & 0.010 \\
\hline Median, mo $(95 \% \mathrm{Cl})$ & NR & 20.4 (NA-NA) & NR & \\
\hline 3-year OS, \% (SE) & $68(5)$ & $48(13)$ & $72(6)$ & \\
\hline missing, $n$ & 9 & 2 & 7 & \\
\hline
\end{tabular}

Abbreviations: CR, complete remission; n, numbers; RFS, relapse-free survival; EFS, event-free survival; OS, overall survival; mo, months; NA: not available; $P, p$-value; NR, not reached; SE, standard error. $P$-value is from Fisher's exact test for $\mathrm{CR}$ rate and from the log-rank test for survival end-points; Statistically significant results are highlighted in bold font. *, including an early death case during induction.

\section{Figures}




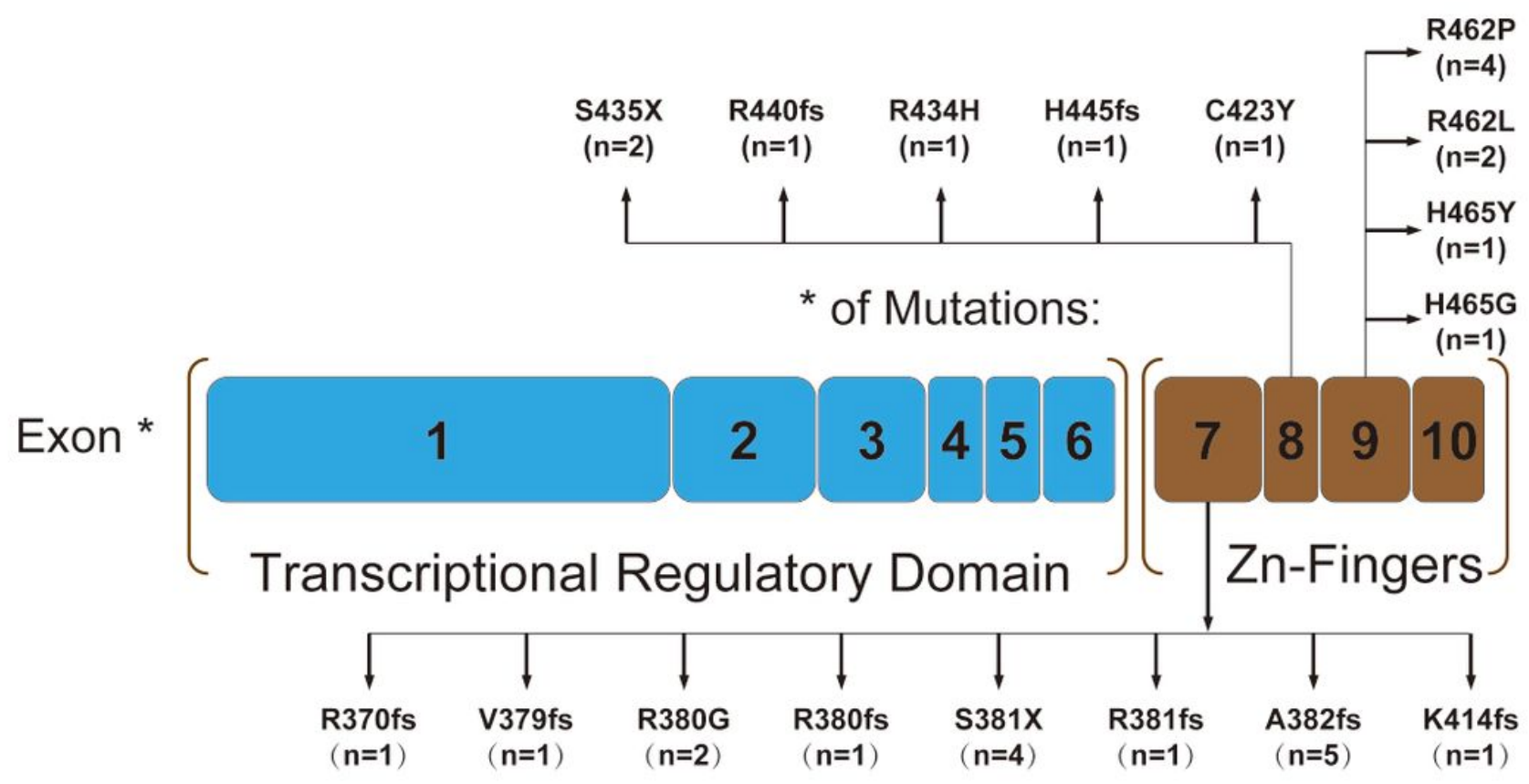

Figure 1

Schematic showing the locations of WT1 mutations found in CEBPA mutated AML. One patient had both the R380G and S381fs mutations.

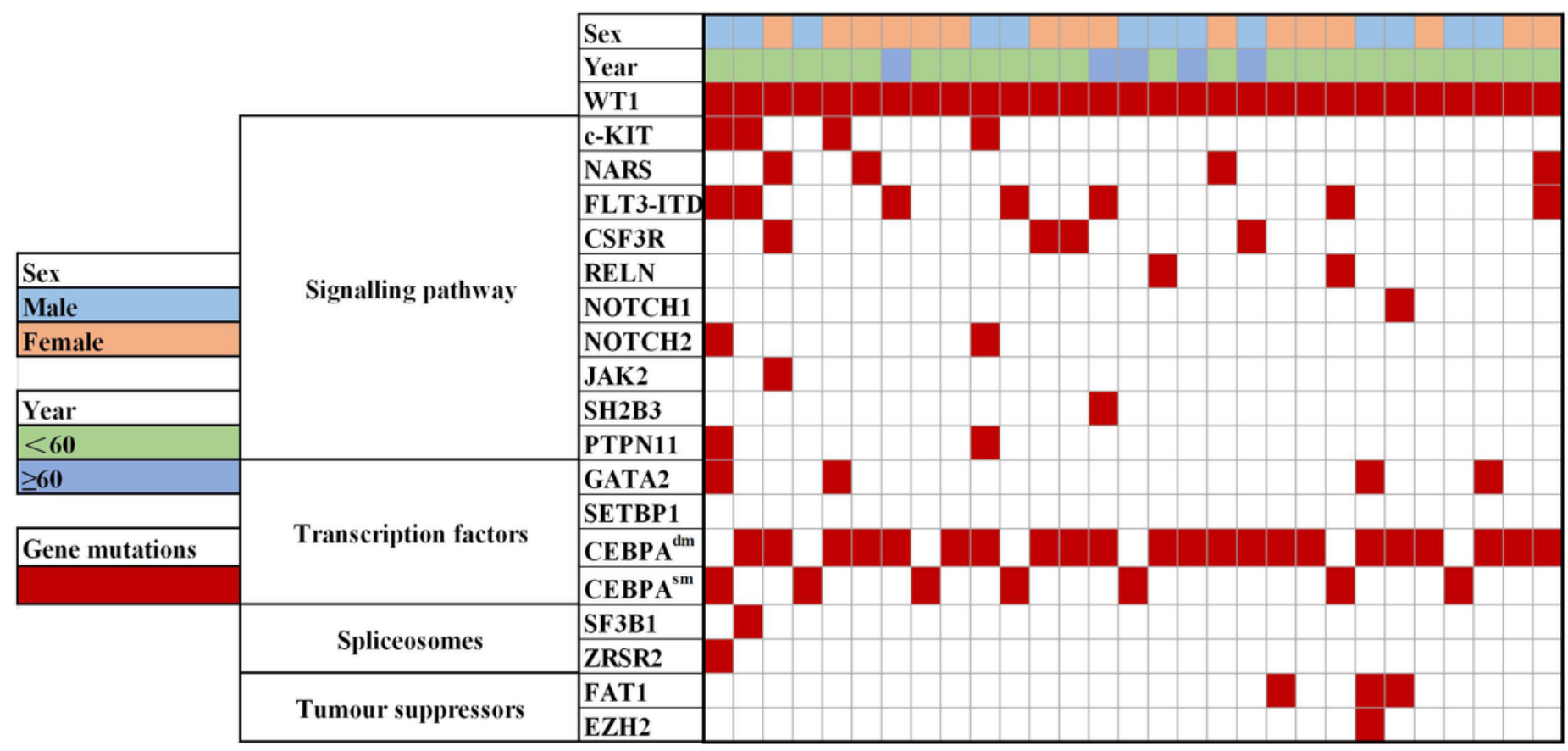

Figure 2 
Spectrum of acquired co-mutations in 29 WT1 mutated patients with CEBPA mutated AML divided into different mutational categories. Each bar represents a distinct driver gene.
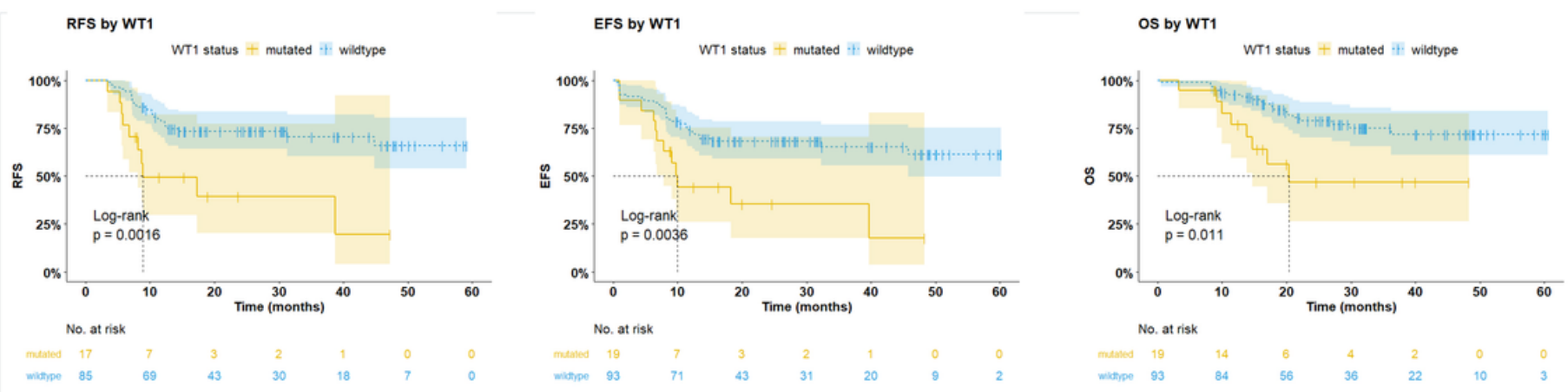

\section{Figure 3}

Influence of mutations in WT1 on survival. a. Kaplan-Meier estimates of Relapse-free survival (RFS) , b. event-free survival (EFS) and c. overall survival (OS) in newly diagnosed patients with CEBPA double mutated AML, according to the presence or absence of mutations in WT1.

\section{Supplementary Files}

This is a list of supplementary files associated with this preprint. Click to download.

- Supplementaltable1.doc 